\title{
Estimating Additive Interaction Effect in Stratified Two-Phase Case-Control Design
}

\author{
$\mathrm{Ai} \mathrm{Ni}^{\mathrm{a}}$ Jaya M. Satagopan ${ }^{\mathrm{b}}$ \\ a Division of Biostatistics, The Ohio State University, Columbus, OH, USA; ${ }^{\mathrm{b}}$ Department of Biostatistics and \\ Epidemiology, School of Public Health, Rutgers University, Piscataway, NY, USA
}

\section{Keywords}

Additive interaction · Inverse-probability weighting ·

Multiple imputation - Offset - Stratified two-phase

case-control design

\begin{abstract}
Background and Aims: There is considerable interest in epidemiology to estimate an additive interaction effect between two risk factors in case-control studies. An additive interaction is defined as the differential reduction in absolute risk associated with one factor between different levels of the other factor. A stratified two-phase case-control design is commonly used in epidemiology to reduce the cost of assembling covariates. It is crucial to obtain valid estimates of the model parameters by accounting for the underlying stratification scheme to obtain accurate and precise estimates of additive interaction effects. The aim of this paper is to examine the properties of different methods for estimating model parameters and additive interaction effects under a stratified two-phase case-control design. Methods: Using simulations, we investigate the properties of three existing methods, namely stratum-specific offset, inverseprobability weighting, and multiple imputation for estimating model parameters and additive interaction effects. We
\end{abstract}

\section{KARGER}

๑ 2019 S. Karger AG, Basel

E-Mail karger@karger.com

www.karger.com/hhe also illustrate these properties using data from two published epidemiology studies. Results: Simulation studies show that the multiple imputation method performs well when both the true and analysis models are additive (i.e., does not include multiplicative interaction terms) but does not provide a discernible advantage over the offset method when the analysis models are non-additive (i.e., includes multiplicative interaction terms). The offset method exhibits the best overall properties when the analysis model contains multiplicative interaction effects. Conclusion: When estimating additive interaction between risk factors in stratified two-phase case-control studies, we recommend estimating model parameters using multiple imputation when the analysis model is additive, and we recommend the offset method when the analysis model is non-additive.

(c) 2019 S. Karger AG, Basel

\section{Introduction}

Stratified two-phase case-control design is a popular sampling strategy to measure new risk factors in a costefficient manner in epidemiology studies [1-3]. A parent cohort is first established, and certain risk factors of interest are ascertained from all subjects. The parent cohort
Ai Ni

Division of Biostatistics, The Ohio State University

1841 Neil Avenue, 280D Cunz Hall

Columbus, OH 43210 (USA)

E-Mail ni.304@ osu.edu 
may be assembled in a prospective or retrospective manner. Next, affected cases and unaffected controls are subsampled from within strata defined by one or more risk factors measured in the parent cohort, and new risk factors are measured only for individuals in the subsample $[4,5]$. This paper investigates the properties of three methods for estimating an interaction between two risk factors on the additive scale of the outcome under a stratified two-phase case-control design.

Epidemiology studies use statistical models to investigate interactions between two (or more) risk factors - for example, interaction between genetic and environmental factors [6], genetic and demographic factors [7], multiple genetic [8], or non-genetic [9] factors. There is considerable enthusiasm in studying interactions because it is anticipated that interactions can shed light on biological mechanisms underlying disease etiology and provide insights into the benefits of changes or behavioral modifications to one risk factor in subgroups of individuals defined by another risk factor [10]. In statistical models, interaction between two risk factors is defined as departure from additivity of the effects of the risk factors, i.e., the effect of one risk factor on the outcome varies across the levels of another risk factor [11]. An interaction is represented by a product term of the risk factors under a canonical link function and is commonly referred to as multiplicative interaction [12]. For binary outcomes, the canonical link function is the logistic link. A multiplicative interaction between two risk factors is the ratio of two ORs, where the numerator is the OR measuring the association between two risk factors in affected individuals (equivalently, individuals having value 1 for the outcome) and the denominator is a similar OR in unaffected individuals (equivalently, individuals having value 0 for the outcome) [13].

Epidemiology studies increasingly examine additive interaction between risk factors, which is defined as differential reduction in absolute risk associated with changing the level of one risk factor across the levels of another risk factor [14-18]. Additive interactions are anticipated to be more useful than multiplicative interactions since they can be directly interpreted in terms of risk reduction [12], and conceptual models for biological interactions between risk factors translate to interactions on an additive scale $[8,19]$.

There is a large and growing body of literature on statistical methods for estimating the additive effects of risk factors and their multiplicative interaction effects in logistic regression models under stratified two-phase designs with retrospective sampling of cases and controls in the first phase. These methods include maximum likelihood estimation with an offset and the weighted likelihood approach [3, 20-27]. To our knowledge, limited attention has been given to statistical evaluations of the properties of these methods for estimating additive interaction effects between risk factors under stratified twophase sampling. This gap exists despite growing interest in the evaluation of additive interactions in epidemiology studies and despite the increasing availability of large epidemiology cohorts that can be used for investigating novel additive interactions between risk factors in a costefficient manner.

Multiple imputation approach has been proposed to estimate the parameters of a logistic regression model under two-phase designs [28-30]. It is a popular approach for obtaining complete data sets in the presence of missing variables in medical studies and is easy to implement [30]. Under this approach, individuals who are not sampled into phase two of the study are treated as having missing value for the new variable measured in the second phase. The missing values are replaced with plausible values of the new variable. The resulting data set is then treated as a complete data set, and a standard logistic regression model is applied to estimate the model parameters from the complete data set. Multiple imputation methods applied to stratified two-phase case-control studies have shown that this approach provides parameter estimates with less bias and better precision than methods based on conditional maximum likelihood estimation [30]. These investigations have focused on estimating the parameters of an additive logistic regression model (i.e., a model without multiplicative interaction terms) and have not examined the properties of estimating additive interactions between risk factors.

In this paper, we examine the properties of three methods, namely, offset, weighted likelihood, and multiple imputation, for estimating additive interaction effects between risk factors in a stratified two-phase design. We provide an empirical illustration of these methods using data from two published epidemiology studies and use simulations to examine the properties of the methods.

\section{Motivating Examples}

Our paper is motivated by the following epidemiology studies: - The Study of Nevi in Children (SONIC) is a population-based prospective study in a US cohort of children to examine risk factors associated with nevus phenotypes [31]. Several putative risk factors including demographic factors and sun exposure were measured on the initial parent cohort of $n=443$ 
subjects during 2004. A main outcome of interest is high-risk nevus phenotype (also referred to as mole-prone phenotype), a binary outcome defined as nevus counts exceeding a certain value. Following prior work in SONIC that defined high-risk phenotype based on quintiles of nevus counts observed in the study subjects [32], in this paper we consider high-risk nevus phenotype as our binary outcome. The parent cohort has since been expanded with prospective ascertainment of more study subjects from different age groups. This prospective cohort is now amenable to future studies of novel risk factors in a cost-efficient manner via a two-phase stratified sampling approach.

- The Epidemiology of Endometrial Cancer Consortium (E2C2) is a large population-based case-control study investigating risk factors associated with the etiology of endometrial cancer [7]. Data on age, body mass index, and genotype at marker rs727479, a single nucleotide polymorphism in the CYP19A1 gene, are available for the parent cohort of 4,261 endometrial cancer cases and 7,099 unaffected controls from tables published by Setiawan et al. [7]. This cohort is also amenable to future cost-efficient studies of novel risk factors via a two-phase stratified sampling approach.

We will use data from SONIC and E2C2 to illustrate statistical methods for estimating additive interactions under a two-phase stratified case-control design. We will estimate additive interaction between sun sensitivity index (a demographic factor) and sun exposure in relation to high-risk nevus phenotype in SONIC, and additive interaction between body mass index and rs727479 in relation to the risk of endometrial cancer in E2C2. The availability of complete data from the parent cohorts in these studies allows us to illustrate the properties of analysis methods for estimating additive interactions by presenting an analysis of the full cohorts along with a side-by-side illustration of analysis based on stratified two-phase sampling applied to these studies.

\section{Notations}

We use the following notations throughout this paper:

1. Let $(D, G, E, X)$ be a vector of observations, where $D$ is a binary outcome denoting a case (i.e., affected status, $D=1$ ) or a control (i.e., unaffected status, $D=0$ ), $G$ and $E$ are risk factors of interest, and $X$ is a vector of other covariates including confounders. In this paper, we take $G$ to be a continuous or categorical risk factor and $E$ to be a categorical risk factor. When discussing the methodological concepts, we focus on $E$ having two categories ( $E=0$ or 1 ). Note that $G$ and $E$ can be any two risk factors of interest and need not be restricted to genetic and environmental factors.

2. Denote $p=\operatorname{pr}(D=1 \mid G, E, X)$ as disease risk given $G, E$, and $X$. We assume that the association between $D$ and $(G, E, X)$ is described by the logistic regression model [33]:

$$
\log \left(\frac{p}{1-p}\right)=\beta_{0}+\beta_{1} G+\beta_{2} E+\beta_{3} G E+\beta_{4} X,
$$

where $\beta_{0}$ is the intercept, $\beta_{1}$ and $\beta_{2}$ are referred to as the additive effects of $G$ and $E$, respectively, $\beta_{3}$ is their non-additive effect, and $\beta_{4}$ is a vector of additive effects for $X$.
3. Denote

$\beta=\left\{\beta_{j}\right\}_{j=1}^{4}$

as the vector of model parameters.

4. Consider a parent data set of $N$ independent individuals. We assume that $D$ and $G$ are measured on these $N$ subjects. In practice, some components of $X$ may also be available for these $N$ subjects. However, in this paper, we assume only $D$ and $G$ are available for the entire parent data set. We further assume that there are $N_{1}$ individuals with $D=1$ (cases) and $N_{0}=N-N_{1}$ individuals with $D=0$ (controls) in the parent data set.

5. Divide the parent data set into $H$ strata using $G$. Let $N_{h}$ denote the sample size of stratum $h=1, \ldots, H$. Clearly,

$N=\sum_{h=1}^{H} N_{h}$.

6. Subsample $n_{h}$ contains individuals from stratum $h$. Let $n=\sum_{h=1}^{H} n_{h}$

denote the size of the subsampled data set. Measure $E$ and $X$ on these $n$ individuals.

7. Denote $\left(D_{h i}, G_{h i}, E_{h i}, X_{h i}, S_{h i}\right)$ as the observations corresponding to person $i\left(=1, \ldots, N_{h}\right)$ in stratum $h(=1, \ldots, H)$. Here, $S_{h i}$ is a sampling indicator with $S_{h i}=1$ if the person is in the subsample and, hence, has a measured $E_{h i}$ and $X_{h i}$; otherwise, $S_{h i}=0$ and $E_{h i}$ and $X_{h i}$ are unmeasured.

8. We use the hat notation to indicate estimated values, e.g., $\hat{\beta}$ is an estimate of $\beta$.

\section{Interaction}

Denoting $f($.) as an arbitrary function (or scale) of disease risk, we define the effect of $E$ when $X$ and $G$ take values $X=x$ and $G=$ $g$ as the contrast

$$
f(p \mid G=g, E=1, X=x)-f(p \mid G=g, E=0, X=x) .
$$

The statistical interaction between $G$ and $E$, evaluated at $X=x$, is a contrast defined as the difference between the effects of $E$ under two values of $G$, namely $G=g_{1}$ and $G=g_{2}$. In notations, the interaction effect is a contrast [34], denoted $A\left(x, g_{1}, g_{2}\right)$, given by

$$
\begin{aligned}
& A\left(x, g_{1}, g_{2}\right) \\
& =\left\{f\left(p \mid G=g_{2}, E=1, X=x\right)-f\left(p \mid G=g_{2}, E=0, X=x\right)\right\} \\
& -\left\{f\left(p \mid G=g_{1}, E=1, X=x\right)-f\left(p \mid G=g_{1}, E=0, X=x\right)\right\} .
\end{aligned}
$$

\section{Multiplicative Interaction}

A multiplicative interaction between $G$ and $E$ is obtained by defining $f($.$) on the logistic scale as f(p \mid G, E, X)=\log \{p /(1-p)\}$. It then follows from equations 1 and 2 that the multiplicative interaction effect is $A\left(x, g_{1}, g_{2}\right)=\beta_{3}\left(g_{2}-g_{1}\right)$, the non-additive effect in equation 1.
$\mathrm{Ni} /$ Satagopan 
Additive Interaction

An additive interaction between $G$ and $E$ is obtained on the risk scale by setting $f(p \mid G, E, X)=\operatorname{pr}(D=1 \mid G, E, X)$, and it is given by

$$
\begin{aligned}
& A\left(x, g_{1}, g_{2}\right) \\
& =\left\{\operatorname{pr}\left(D=1 \mid G=g_{2}, E=1, X=x\right)-\operatorname{pr}\left(D=1 \mid G=g_{2}, E=0, X=x\right)\right\} \\
& -\left\{\operatorname{pr}\left(D=1 \mid G=g_{1}, E=1, X=x\right)-\operatorname{pr}\left(D=1 \mid G=g_{1}, E=0, X=x\right)\right\} .
\end{aligned}
$$

The components $\operatorname{pr}(D=1 \mid G, E, X)$ are disease risks, which can be estimated from equation 1 . We can estimate the additive interaction by plugging in the estimated parameters of equation 1 to calculate disease risk, and we derive its variance using the delta method [33].

The components of the right-hand side of equation 3 can be obtained directly from the estimated model parameters in prospective samples such as the SONIC study. However, it is not straightforward to estimate disease risks in this manner from retrospective samples such as the E2C2 study, since the intercept $\beta_{0}$ cannot be interpreted in terms of baseline risk in the population. Therefore, it is common to report the relative excess risk due to interaction (RERI) as a measure of additive interaction in the analysis of retrospective samples $[18,19]$, which is defined as the ratio $A\left(x, g_{1}, g_{2}\right) / \operatorname{pr}\left(D=1 \mid G=g_{1}, E=0, X=x\right)$. For rare diseases, RERI can be approximated as $\exp \left\{\beta_{1}\left(g_{2}-g_{1}\right)+\beta_{2}+\beta_{3} g_{2}\right\}-\exp \left\{\beta_{1}\left(g_{2}-\right.\right.$ $\left.\left.g_{1}\right)\right\}-\exp \left\{\beta_{2}+\beta_{3} g_{1}\right\}+1$, which does not depend upon the baseline parameter $\beta_{0}$ and the effect $\beta_{4}$ of $X$.

\section{Stratified Design}

Denote $N_{h 1}$ and $N_{h 0}$ as the known number of cases and controls, respectively, in stratum $h(=1, \ldots, H)$. Subsample $n_{h 1}$ cases and $n_{h 0}$ controls are from stratum $h$ using sampling probabilities $\pi_{h 1}$ and $\pi_{h 0}$, respectively, where $\pi_{h d}=n_{h d} / N_{h d}, d=0,1, h=1, \ldots, H[2]$. Without loss of generality, we assume that we select all cases and an equal number of controls from each stratum. Therefore, $n_{h 1}=$ $N_{h 1}, n_{h 0}=\min \left(N_{h 0}, N_{h 1}\right), \pi_{h 1}=1$, and $\pi_{h 0}=\min \left(1, N_{h 1} / N_{h 0}\right)$. Note that when there are more cases than controls in a given stratum, all cases and controls are selected. The total number of individuals subsampled from stratum $h$ is $n_{h}=n_{h 1}+n_{h 0}$. The total numbers of cases and controls in the stratified case-control subset are

$$
n_{1}=\sum_{h=1}^{H} n_{h 1}
$$

and

$$
n_{0}=\sum_{h=1}^{H} n_{h 0}
$$

respectively. Additional risk factors such as $E$ are measured in these $n=n_{1}+n_{0}$ individuals.

Our goal is to estimate the additive interaction between $G$ and $E$ given by equation 3 and its SE using data from the stratified subsample. In the following section, we summarize three existing methods for obtaining consistent estimates of $\beta$ under a stratified sampling design, and we use these estimates to calculate the additive interaction and its SE.

\section{Estimation Methods}

\section{Stratum-Specific Offset}

The disease risk of person $i$ selected into stratum $h$ is conditioned on the sampling indicator $S_{h i}=1$. Hence, the disease risk for person $i$ is given by $\operatorname{pr}\left(D_{h i}=1 \mid S_{h i}=1, G_{h i}, E_{h i}, X_{h i}\right)$.

It follows from Bayes' theorem that

$$
\begin{aligned}
& \operatorname{pr}\left(D_{h i}=1 \mid S_{h i}=1, G_{h i}, E_{h i}, X_{h i}\right) \\
& =\frac{\operatorname{pr}\left(S_{h i}=1 \mid D_{h i}=1, G_{h i}, E_{h i}, X_{h i}\right) \operatorname{pr}\left(D_{h i}=1 \mid G_{h i}, E_{h i}, X_{h i}\right)}{\sum_{d=0}^{1} \operatorname{pr}\left(S_{h i}=1 \mid D_{h i}=d, G_{h i}, E_{h i}, X_{h i}\right) \operatorname{pr}\left(D_{h i}=d \mid G_{h i}, E_{h i}, X_{h i}\right)} \\
& =\frac{\pi_{h 1} \exp \left(\mu_{h i}\right)}{\pi_{h 1} \exp \left(\mu_{h i}\right)+\pi_{h 0}} \\
& =\frac{\exp \left\{\log \left(\pi_{h 1} / \pi_{h 0}\right)+\mu_{h i}\right\}}{1+\exp \left\{\log \left(\pi_{h 1} / \pi_{h 0}\right)+\mu_{h i}\right\}},
\end{aligned}
$$

where $\mu_{h i}=\log \left\{\operatorname{pr}\left(D_{h i}=1 \mid G_{h i}, E_{h i}, X_{h i}\right) / \operatorname{pr}\left(D_{h i}=0 \mid G_{h i}, E_{h i}, X_{h i}\right)\right\}$ is the linear predictor from a logistic model based on a prospective sample.

Therefore, the log-likelihood function of the stratified casecontrol subsample is

$$
\ell(\beta)=\sum_{h=1}^{H} \sum_{i=1}^{n_{h}} \log \left\{\operatorname{pr}\left(D_{h i} \mid S_{h i}=1, G_{h i}, E_{h i}, X_{h i} ; \beta\right)\right\},
$$

where the risks on the right-hand side are given by equation 4 . By including $\log \left(\pi_{h 1} / \pi_{h 0}\right)$ as an offset for stratum $h$, we can obtain $\hat{\beta}$ as consistent estimates of $\beta$ by maximizing equation 5 . Since $n_{h 1}=$ $N_{h 1}$, the offset simplifies to $\log \left(1 / \pi_{h 0}\right)=\max \left\{\log \left(N_{\mathrm{h} 0} / N_{h 1} / h_{1}\right), 0\right\}$. The variance of $\hat{\beta}$ is provided by [35] and [36], and it is summarized in the Appendix.

\section{Inverse-Probability Weighting}

For the stratified case-control sample, denote

$$
U_{h d i}(\beta)=\frac{\partial}{\partial \beta} \log \left\{\operatorname{pr}\left(D_{h i}=d \mid G_{h i}, E_{h i}, X_{h i}\right)\right\}
$$

as the logistic score function corresponding to person $i(=1, \ldots$, $\left.n_{h d}\right)$ with disease status $d(=0,1)$ in stratum $h(=1, \ldots, H)$. We can obtain the inverse-probability weighted (IPW) estimate $\hat{\beta}$ as a solution to

$$
U(\beta)=\sum_{d=0}^{1} \sum_{h=1}^{H} \frac{1}{\pi_{h d}} \sum_{i=1}^{n_{h d}} U_{h d i}(\beta)=0 .
$$

It can be shown that the IPW estimate of $\beta$ is consistent [37]. Its variance can be estimated as given by Breslow and Chatterjee [3]

$$
\left(\frac{\partial U}{\partial \beta^{T}}\right)^{-1} \sum_{d=0}^{1} \sum_{h=1}^{H} \pi_{h d}^{-2}\left\{\sum_{i=1}^{n_{h d}} U_{h d i}^{\otimes 2}-\frac{1-\pi_{h d}}{n_{h d}}\left(\sum_{i=1}^{n_{h d}} U_{h d i}\right)^{\otimes 2}\right\}\left(\frac{\partial U}{\partial \beta}\right)^{-1},
$$

where the notation $a^{\otimes 2}$ denotes $a a^{T}$.

\section{Multiple Imputation}

Since $E$ and $X$ are measured only in the stratified case-control subsample of $n$ individuals, their values in the $N-n$ unselected subjects can be deemed missing. The probability of a subject being selected into the subsample depends only on $D$ and $G$. Therefore, 
the missingness mechanism for $E$ and $X$ can be classified as missing at random [38]. Thus, one can use multiple imputation (MI) to fill in the missing values in the $N-n$ unselected subjects and use the full cohort of $N$ individuals to estimate the model parameters $\beta$ via the standard logistic regression, instead of restricting our analysis to the case-control subsample of $n$ individuals as done under the offset and IPW methods.

Several methods for MI are currently available in the literature [39-41]. In this paper, we use the chained equation method [42], a flexible approach that assumes the existence of the conditional distribution of the missing variable being imputed given all other variables including the outcome in an imputation model [43]. Briefly, the chained equation method starts with some initial values for missing data and imputes missing variables one at a time in a cyclical fashion by sampling from their conditional distributions given all other variables in the imputation model. This procedure is iterated until convergence [42]. In this method, the conditional distribution of categorical variables being imputed, given all other variables in the imputation model, is assumed to be binomial or multinomial; the conditional distribution of continuous variables being imputed, given all other variables in the imputation model, is assumed to be normal. One can choose any suitable imputation method to impute individual missing variables. An important requirement for the imputation model is that it has to be at least as saturated (in terms of the number of parameters) as the analysis model to be fitted to the imputed data [41, 44].

The imputed values are used to estimate the model parameters and the additive interaction of interest. This entire iterative procedure is carried out $m$ times (typically $m=5-10$ ) to obtain multiple imputed data sets and the corresponding additive interaction estimates. The final estimate of additive interaction is then obtained as the empirical average of these estimates. Its variance is estimated by Rubin's rule, $\operatorname{var}(A)=W+(1+1 / m) B$, where $W$ is the within-imputation variance and $B$ is the between-imputation variance. Readers are referred to Rubin [38] for details. In all our illustrative examples and simulations, we used $m=10$ imputed data sets.

\section{Software for $M I$}

The chained equation method is implemented in the mice (multiple imputation by chained equation) function in the R programming language and in the FCS (fully conditional specification) statement in the PROC MI procedure in the SAS software.

\section{Illustrative Examples}

In this section, we provide empirical illustration of estimating additive interactions and their SEs via the above parameter estimation methods using data from two published cancer epidemiology studies.

\section{Study of Nevi in Children}

Nevi are among the important known risk factors for melanoma and are anticipated to be associated with magnitude of sun sensitivity and sun exposure even in children. SONIC (the Study of Nevi in Children) is the first
Table 1. Participant characteristics $(n=443)$ of the SONIC study

\begin{tabular}{lc}
\hline Male sex, $n(\%)$ & $193(43.6)$ \\
White race, $n(\%)$ & $203(45.8)$ \\
Sun exposure, $n(\%)$ & \\
$\quad$ Low & $114(25.7)$ \\
$\quad$ Medium & $129(29.1)$ \\
$\quad$ High & $77(17.4)$ \\
Mean SSI \pm SD & $1.66 \pm 0.78$ \\
\hline
\end{tabular}

SSI, sun sensitivity index.

known population-based prospective study of nevi in a US cohort of children to evaluate risk factors for nevi [31]. The parent data set includes $n=443$ prospectively ascertained children with the following measurements: the total number of nevi on the back (obtained using imaging techniques), sex and race/ethnicity (obtained from school records), sun sensitivity index (SSI, a continuous score obtained by SONIC investigators by combining numerical values assigned to skin color, hair color and Fitzpatrick tendency to burn, which were recorded by a school nurse), and sun exposure (obtained from a questionnaire).

As described in the Motivating Examples section above, the binary outcome is high-risk nevus phenotype, defined as presence or absence of nevus counts in the upper quintile of the observed counts [32]. A total of 91 individuals had high-risk nevus phenotype (cases). The covariate vector $X$ consists of two binary variables: sex (male or female) and race/ethnicity (non-Hispanic White or other). The continuous risk factor $G$ is SSI, which takes values between 0 (high sun sensitivity) and 3 (low sun sensitivity). The risk factor $E$ is sun exposure, an ordinal variable denoting low ( $\leq 2 \mathrm{~h}$ per day, on average, spent outdoor during summer), medium (3-4 h spent outdoors) and high ( $>4 \mathrm{~h}$ spent outdoors) exposure. Table 1 summarizes the characteristics of all 443 individuals in the parent SONIC cohort.

We stratified the parent data set on SSI using steps of 0.2 units. When a stratum had no case, we combined it with an adjacent stratum. We obtained $H=14$ strata as shown in Appendix Table A1. We obtained a stratified case-control subsample by sampling all cases and, where feasible, an equal number of controls from each stratum, yielding $n_{1}=91$ cases and $n_{0}=87$ controls $(n=178)$. In our set up, $D$ and SSI are measured on all 443 individuals in the parent data set, and $E$ and $X$ are measured only in the subsample of 178 children. 
Table 2. Estimated regression coefficients (and standard errors in parenthesis) from the SONIC study data

\begin{tabular}{|c|c|c|c|c|c|}
\hline Intercept & $-3.95(0.73)$ & $-3.41(0.78)$ & $-5.16(0.70)$ & $-5.21(0.83)$ & $-3.51(0.83)$ \\
\hline Sun exposure (high vs. low) & $0.34(0.37)$ & $0.35(0.67)$ & $0.27(0.50)$ & $0.18(0.57)$ & $0.20(0.50)$ \\
\hline $\mathrm{SSI}^{*}$ & $1.32(0.72)$ & $1.44(0.74)$ & $5.60(0.65)$ & $5.33(0.96)$ & $3.25(0.91)$ \\
\hline $\mathrm{SSI}^{2}$ & $-0.79(0.31)$ & $-0.83(0.31)$ & $-2.62(0.29)$ & $-2.48(0.41)$ & $-1.33(0.39)$ \\
\hline
\end{tabular}

Full cohort, analysis on the full cohort; MI, multiple imputation on the stratified case-control sample; Offset, offset method on the stratified case-control sample; IPW, inverse-probability weighting method on the stratified case-control sample; Naive, naive logistic regression on the stratified case-control sample; SSI, sun sensitivity index. * Stratifying variable.

We fitted a logistic regression model for the binary outcome with respect to SSI, sun exposure, race/ethnicity, and sex. We included two indicator variables $E_{\text {high }}$ and $E_{\text {med }}$ for sun exposure, representing high and medium exposures, respectively (taking low exposure as the reference category). Prior works have shown a quadratic effect of SSI and no interaction between SSI and sun exposure in relation to nevus counts [32]. Building on this work, we fitted the following logistic regression model to the stratified case-control subsample:

$$
\begin{aligned}
& \log \left(\frac{p}{1-p}\right) \\
& =\beta_{0}+\beta_{1} S S I+\beta_{2} S S I^{2}+\beta_{3} E_{\text {med }}+\beta_{4} E_{\text {high }}+\beta_{5} \text { race }+\beta_{6} \text { sex. }
\end{aligned}
$$

Table 2 shows the results. Columns 3, 4, and 5 show the parameter estimates based on the MI, offset, and IPW methods using the 178 stratified case-control subsamples. As a benchmark for comparison, Column 2 shows the analysis of the full parent data set of all 443 individuals. As another benchmark, Column 6 shows the results of a naive logistic regression approach that analyzes the second phase data using standard logistic regression, ignoring the stratified sampling scheme. The offset, IPW, and naive methods give considerably inflated estimates of the (linear and quadratic) effects of the stratification variable SSI. Overall, the MI method gives estimates that are closer to those based on the full cohort.

Nevi are not rare events. Hence, RERI cannot be approximated using the rare disease assumption as shown in the Additive Interaction section. Further, SONIC is a prospective study. Therefore, we estimated additive interaction using equation 3 instead of estimating RERI. We used the parameter estimates obtained from the above logistic regression model and estimated the additive interaction between SSI and sun exposure among non-Hispanic White male children with SSI $=1\left(=g_{1}\right)$ and $2.5\left(=g_{2}\right)$ and medium $(E=1)$ and low $(E=0)$ sun exposure levels. The results, shown in Table 3, illustrate the effect of the different parameter estimation methods on estimating the additive interaction effect. The MI method gives risk differences and additive interaction estimates close to those obtained using the full cohort. Additive interaction estimates using the naive method are considerably smaller than those of the full cohort. The offset method gives risk differences and additive interaction estimates closer to the full cohort than the MI method. However, the SE of the additive interaction between SSI and sun exposure is slightly larger using the offset method rather than the MI method. These results suggest that the offset and MI methods have similar performances in terms of estimating additive interaction, although the MI method had a better performance for estimating the individual model parameters.

Using the MI approach, the risk differences between low and medium sun exposure for non-Hispanic White children were 10.6 and $3.0 \%$ at SSI $=1$ and 2.5 , respectively. This suggests that a greater risk reduction can be obtained by reducing sun exposure from medium to low levels for children with high (SSI =1) than low (SSI = 2.5) sun sensitivity. The estimated additive interaction was 7.6\% (SE $=9.0 \%)$, suggesting that non-Hispanic White children with high sun sensitivity can gain $7.6 \%$ more risk reduction than those with low sun sensitivity by reducing their sun exposure from medium to low level. We obtained similar results for additive interactions obtained by comparing high and low sun exposure groups. 
Table 3. Estimated additive interactions between SSI and sun exposure and their standard errors in SONIC

\begin{tabular}{lllllr}
\hline & Full cohort & MI & Offset & IPW & Naive \\
\hline Medium versus low sun exposure & & & & & 0.069 \\
Risk difference in SSI = 1 & 0.137 & 0.106 & 0.107 & 0.071 & 0.087 \\
Risk difference in SSI = 2.5 & 0.043 & 0.030 & 0.005 & 0.003 & -0.018 \\
Additive interaction (SSI 1 vs. 2.5) & 0.093 & 0.076 & 0.102 & 0.068 & 0.026 \\
Standard error of additive interaction & 0.062 & 0.090 & 0.10 & 0.120 & 0.123 \\
\hline High versus low sun exposure & & & & & 0.136 \\
Risk difference in SSI = 1 & 0.270 & 0.213 & 0.191 & 0.008 & 0.185 \\
Risk difference in SSI = 2.5 & 0.110 & 0.082 & 0.014 & 0.128 \\
Additive interaction (SSI 1 vs. 2.5) & 0.160 & 0.130 & 0.177 & 0.128 \\
Standard error of additive interaction & 0.091 & 0.15 & 0.150 & 0.210 & 0.090 \\
\hline
\end{tabular}

Full cohort, analysis on the full cohort; MI, multiple imputation on the stratified case-control sample; Offset, offset method on the stratified case-control sample; IPW, inverse-probability weighting method on the stratified case-control sample; Naive, naive logistic regression on the stratified case-control sample; SSI, sun sensitivity index.

\section{Endometrial Cancer Study}

E2C2 [45] is a population-based retrospective casecontrol study that examines risk factors associated with the etiology of endometrial cancer. Setiawan et al. [7] reported the following data from 4,261 endometrial cancer cases and 7,099 unaffected controls ascertained in a retrospective manner: age (binary: $<55$ and $\geq 55$ years), body mass index (BMI; 3 categories: $<25 \mathrm{~kg} / \mathrm{m}^{2}$ or normal, $25-$ $30 \mathrm{~kg} / \mathrm{m}^{2}$ or overweight, and $\geq 30 \mathrm{~kg} / \mathrm{m}^{2}$ or obese), and genotypes at marker rs727479, a single nucleotide polymorphism (SNP), in the gene CYP19A1 (3 genotype categories: $A A, A C$, and $C C)$. This is our parent data set of $n=11,360(4,261+7,099)$ individuals. This data set is given in Table 4 of the paper by Setiawan et al. [7], and is, hence, not tabulated again in this paper. We stratified the parent data on BMI, which has $H=3$ strata, and drew case-control subsamples from the 3 strata by sampling all cases and, where feasible, an equal number of controls. This gave us 4,261 cases and 4,111 controls in the stratified case-control subsample. For illustrating the properties of additive interaction estimated via the offset, IPW, and MI methods under a stratified two-phase design, we assumed that BMI and age were measured on all subjects in the parent data set, and the genotypes at marker rs727479 were measured only in the subsample.

Setiawan et al. [7] fitted separate models to the $<55$ and $\geq 55$ age groups and included an interaction between BMI and the SNP in each model. Following their work, we fitted the following logistic regression model, separately to the two age groups:

$$
\begin{aligned}
& \log \left(\frac{p}{1-p}\right) \\
&=\beta_{0}+\beta_{1} S N P_{A C}+\beta_{2} S N P_{A A}+\beta_{3} B M I_{\text {overweight }}+\beta_{4} B M I_{\text {obese }}+ \\
& \beta_{5} S N P_{A C} \times B M I_{\text {overweight }}+\beta_{6} S N P_{A A} \times B M I_{\text {overweight }}+ \\
& \beta_{7} S N P_{A C} \times B M I_{\text {obese }}+\beta_{8} S N P_{A A} \times B M I_{\text {obese }}
\end{aligned}
$$

where $p$ denotes the risk of endometrial cancer given $\mathrm{BMI}$ and genotypes of the SNP rs727479, $S N P_{g}$ is an indicator variable taking value 1 if the genotype is $g$ (= AA or $\mathrm{AC}$ ) and taking value 0 otherwise. Similarly, $B M I_{b}$ is an indicator variable taking value 1 if $\mathrm{BMI}$ is $b$ (= overweight or obese) and taking value 0 otherwise. Thus, the CC genotype category and the normal BMI category are reference groups. Since the imputation model needs to be as saturated (in terms of the number of parameters) as the analysis model 9 [41, 44], we allowed interaction between genotype and BMI in the MI model by performing separate MI within each BMI level.

Table $4 \mathrm{a}$ and $\mathrm{b}$ show the parameter estimates under the various estimation methods for age $\geq 55$ and $<55$ groups, respectively. The results based on the full parent data set and a naive analysis of the subsample are also shown as benchmarks for comparison. As before, the naive approach performs poorly in estimating the intercept and the effects of BMI, the stratification factor. The MI, offset, and IPW methods give similar parameter estimates that are also close to estimates obtained under the full cohort approach. These three methods have similar performances possibly because of the large sample size. 
Table 4. Estimated regression coefficients (and standard errors in parenthesis) of the endometrial cancer study data

\begin{tabular}{|c|c|c|c|c|c|}
\hline & Full cohort & MI & Offset & IPW & Naive \\
\hline \multicolumn{6}{|l|}{ a $\quad$ Age $\geq 55$} \\
\hline Intercept & $-0.830(0.100)$ & $-0.829(0.105)$ & $-0.813(0.116)$ & $-0.813(0.118)$ & $0.080(0.121)$ \\
\hline $\mathrm{SNP}_{\mathrm{AC}}$ & $-0.096(0.114)$ & $-0.094(0.121)$ & $-0.111(0.136)$ & $-0.111(0.133)$ & $-0.111(0.136)$ \\
\hline $\mathrm{SNP}_{\mathrm{AA}}$ & $-0.049(0.113)$ & $-0.054(0.119)$ & $-0.072(0.136)$ & $-0.072(0.132)$ & $-0.072(0.136)$ \\
\hline $\mathrm{BMI}_{\text {overweight }}^{*}$ & $0.022(0.169)$ & $0.063(0.184)$ & $0.037(0.191)$ & $0.037(0.193)$ & $-0.318(0.194)$ \\
\hline $\mathrm{SNP}_{\mathrm{AA}} * \mathrm{BMI}_{\text {overweight }}$ & $0.396(0.187)$ & $0.346(0.207)$ & $0.375(0.214)$ & $0.375(0.214)$ & $0.375(0.214)$ \\
\hline $\mathrm{SNP}_{\mathrm{AC}} * \mathrm{BMI}_{\text {obese }}$ & $0.498(0.204)$ & $0.496(0.208)$ & $0.512(0.218)$ & $0.512(0.214)$ & $0.512(0.218)$ \\
\hline $\mathrm{SNP}_{\mathrm{AA}} * \mathrm{BMI}_{\text {obese }}$ & $0.619(0.203)$ & $0.624(0.207)$ & $0.642(0.217)$ & $0.642(0.212)$ & $0.642(0.217)$ \\
\hline Additive interaction & 0.152 & 0.153 & 0.157 & 0.157 & 0.160 \\
\hline SE of additive interaction & 0.048 & 0.048 & 0.050 & 0.049 & 0.053 \\
\hline RERI & 1.305 & 1.307 & 1.305 & 1.305 & 0.525 \\
\hline $\mathrm{SNP}_{\mathrm{AC}}$ & $0.221(0.192)$ & $0.270(0.210)$ & $0.267(0.219)$ & $0.267(0.214)$ & $0.267(0.219)$ \\
\hline $\mathrm{SNP}_{\mathrm{AA}}$ & $0.194(0.191)$ & $0.214(0.197)$ & $0.211(0.217)$ & $0.211(0.212)$ & $0.211(0.217)$ \\
\hline $\mathrm{BMI}_{\text {overweight }}^{*}$ & $0.850(0.282)$ & $0.874(0.305)$ & $0.822(0.302)$ & $0.822(0.304)$ & $0.348(0.305)$ \\
\hline $\mathrm{BMI}_{\text {obese }}$ & $0.899(0.305)$ & $0.928(0.310)$ & $0.926(0.318)$ & $0.926(0.316)$ & $0.214(0.319)$ \\
\hline $\mathrm{SNP}_{\mathrm{AC}} * \mathrm{BMI}_{\text {overweight }}$ & $-0.532(0.315)$ & $-0.580(0.349)$ & $-0.526(0.341)$ & $-0.526(0.340)$ & $-0.526(0.341)$ \\
\hline $\mathrm{SNP}_{\mathrm{AA}} * \mathrm{BMI}_{\text {overweight }}$ & $-0.326(0.309)$ & $-0.335(0.331)$ & $-0.274(0.335)$ & $-0.274(0.334)$ & $-0.274(0.335)$ \\
\hline $\mathrm{SNP}_{\mathrm{AC}} * \mathrm{BMI}_{\text {obese }}$ & $-0.014(0.341)$ & $-0.063(0.351)$ & $-0.060(0.357)$ & $-0.060(0.353)$ & $-0.060(0.357)$ \\
\hline $\mathrm{SNP}_{\mathrm{AA}} * \mathrm{BMI}_{\text {obese }}$ & $0.024(0.336)$ & $0.005(0.34)$ & $0.008(0.352)$ & $0.008(0.348)$ & $0.008(0.352)$ \\
\hline Additive interaction & 0.013 & 0.010 & 0.010 & 0.010 & 0.002 \\
\hline SE of additive interaction & 0.080 & 0.080 & 0.082 & 0.081 & 0.087 \\
\hline RERI & 0.384 & 0.381 & 0.383 & 0.383 & 0.068 \\
\hline SE of RERI & 0.727 & 0.750 & 0.752 & 0.752 & 0.419 \\
\hline
\end{tabular}

Additive interactions and their SEs and RERIs and their SEs are shown in the last four rows of each table half. Full cohort, analysis on full cohort; MI, analysis on stratified case-control sample using multiple imputation; Offset, analysis on stratified case-control sample using offset method; IPW, analysis on stratified case-control sample using inverse-probability weighting method; Naive, naive analysis on stratified case-control sample ignoring the design; SE, standard error; RERI, relative excess risk due to interaction; $\mathrm{BMI}_{\mathrm{overweight}}, 25$ $\mathrm{kg} / \mathrm{m}^{2} \leq \mathrm{BMI} \leq 30 \mathrm{~kg} / \mathrm{m}^{2} ; \mathrm{BMI}_{\text {obese }}, \geq 30 \mathrm{~kg} / \mathrm{m}^{2}$. The additive interactions are the differences of the risk difference of obese vs. normal BMI between genotype AA and CC. * Stratifying variable.

We calculated additive interaction between BMI (obese vs. normal groups) and the SNP rs727479 (AA vs. CC genotypes) in the $\geq 55$ and $<55$ age groups separately. These estimates and their SEs, obtained under the various methods, are shown at the bottom of Table $4 \mathrm{a}$ and $\mathrm{b}$ separately. For the $<55$ age group, all three methods - offset, IPW, and MI - give similar additive interaction estimates (estimated additive interaction $=0.010$ units) that are also close to that obtained under the full cohort method ( 0.013 units). The additive interaction estimated under the naive approach (0.002 units) is considerably smaller than that under the full cohort approach. For the $\geq 55$ age group, the estimated additive interaction of the MI method
(0.153 units) is similar to that of the full cohort method (0.152 units). The offset and IPW methods give similar additive interaction estimates ( 0.157 units) that are only slightly larger than that under the MI method. The naive approach has the largest additive interaction estimate of all methods considered ( 0.160 units). The SEs of the additive interaction estimates under the offset, IPW, and MI methods are close to those under the full cohort analysis for both the $<55$ and $\geq 55$ age groups.

It should be noted that the parent cohort of this study was ascertained in a retrospective manner. Therefore, the estimation of additive interaction only serves as an illustration of implementing equation 3 and should not be 
interpreted as an estimation of the true additive interaction in the population. Since the model intercept cannot be interpreted in terms of population-level risk of endometrial cancer and endometrial cancer is rare in the population, we also estimated RERI as a measure of additive interaction for this study. For the $<55$ age group, the offset and IPW methods have similar RERI estimates $(0.383$ units) that are also closer to the full cohort approach (0.384 units) and are slightly larger than RERI estimated under the MI approach ( 0.381 units). The naive approach has considerably smaller RERI ( 0.068 units). For the $\geq 55$ age group, the estimated RERI of the offset and IPW methods (1.305 units) are closer to that of the full cohort method (1.305 units) than that under the MI method (1.307 units). The naive approach gives the smallest additive interaction ( 0.525 units) of all methods considered. The SE of RERI under the MI method is closer to that under the full cohort analysis for both $<55$ and $\geq 55$ age groups.

\section{Numerical Studies}

Using the SONIC data set as a prototype, we conducted simulation studies to evaluate the bias, SEs, and biasvariance trade-off of additive interaction effects estimated under a stratified two-phase design where the parent cohort has binary outcomes and is ascertained in a prospective manner.

We examined the properties where the parameters of the stratified design are estimated via the offset, IPW, and MI methods. Since the naive approach had the worst performance in our empirical illustrations, we excluded this approach from further consideration in our simulation studies. In the remainder of this section, we use the term "additive model" to refer to a logistic regression model containing the additive effects of two risk factors of interest, but not their multiplicative interaction. We use the term "non-additive model" to refer to a logistic regression model containing the additive effects of the risk factors of interest as well as their multiplicative interaction.

In our simulations, the parent data set consisted of $N$ individuals. We considered three variables, denoted $G, E$, and $X$, and simulated a binary outcome for each individual in the parent data set from the following logistic regression model: $\log \{p /(1-p)\}=\beta_{0}+\beta_{1} G+\beta_{2} E+\beta_{3} G \times$ $E+\delta X$, where $p=\operatorname{pr}($ disease $\mid G, E, X)$. Throughout, we considered $E$ to be a binary risk factor of interest with prevalence $P(E=1)=0.40$ and $X$ to be a binary covariate of interest with prevalence $P(X=1)=0.50$. We conduct- ed simulations by first treating $G$ as a continuous risk factor, a uniformly distributed random variable in the range $(0,3)$. We generated $G, E$, and $X$ for each individual from a 3-dimensional zero-mean multivariate normal distribution with unit variance and pair-wise correlation of 0.50 . We derived $G$ from the scaled cumulative distribution of the first component of the multivariate normal distribution, and we obtained $E$ and $X$ by dichotomizing the second and third components, respectively, at appropriate cut points corresponding to the desired prevalence. We also conducted additional simulations by treating $G$ as a categorical variable with two categories and prevalence $P(G=1)=0.50$. We simulated a binary $G$ by dichotomizing the first component of the multivariate normal distribution at the median.

We set $N=500,2,000,3,500$, and $5,000, \beta_{0}=-2.75$ to obtain an expected marginal disease probability of 0.20 (as in the SONIC study illustrated above), $\beta_{2} \in\{0.10$, $0.75\}$ to denote small and large effects of $E$, and $\beta_{3} \in\{0$, $0.50\}$ to denote the absence and presence of a multiplicative interaction between $G$ and $E$. When $G$ was continuous, we set its true effect as $\beta_{1}=0.20$. For binary $G$, we set $\beta_{1}=0.50$. In total, this gave 32 parametric combinations for our simulations. The effect of $X, \delta$, was set to 1 in all scenarios.

We generated 500 data sets under each parametric configuration and analyzed each data set as follows: (i) full cohort design consisting of all $N$ individuals with their observed values of $G, E$, and $X$; and (ii) a stratified two-phase design using $G$ to define the strata and by selecting all cases and an equal number of controls from each stratum. In the stratified design, we assumed that $E$ and $X$ were measured only on individuals ascertained into the subsample. Throughout, we assumed that $G$ was available for all $N$ individuals. When $G$ was a continuous variable, we defined strata by steps of 0.2 units of $G$. When $G$ was a categorical variable, the categories were the strata. Under each design, we analyzed the data set by fitting additive and non-additive models and estimated additive interaction between $G$ and $E$ using equation 3 by setting $x=1, g_{1}=2$, and $g_{2}=3$ for continuous $G$ and $g_{1}=0$ and $g_{2}=1$ for categorical $G$. Under the stratified design, we estimated model parameters via the offset, IPW, and MI methods.

When using MI to estimate parameters from a nonadditive model, we imputed $E$ and $X$ in each stratum separately to ensure that the imputation model was compatible with the analysis model. For continuous $G$, the value of $G$ varied within a given stratum. Hence, we included it in the stratum-specific imputation model. However, for 
categorical $G$, we did not include $G$ in the stratum-specific imputation model since it was constant within each stratum.

We examined the performance of the different methods for estimating the model parameters and additive interaction effects using various summary measures. Our primary measures were: (i) 95\% coverage, defined as the proportion of simulated data sets, out of 500, where the true model parameter or the true additive interaction effect was included within the $95 \% \mathrm{CI}$; and (ii) mean squared error (MSE), defined as the squared difference between the true and estimated values, averaged over 500 data sets under a given parametric configuration. Figures corresponding to these primary measures are included in the main body of this paper. We also examined the following secondary summary measures: (i) bias, defined as the difference between the estimated and true values; (ii) empirical standard error (SEE), defined as the standard deviation of the estimates from the 500 data sets; and (iii) percent bias in SE, defined as the difference between the estimated SE and SEE, divided by SEE. Figures corresponding to these secondary measures are included as online supplementary material (see www.karger.com/doi/10.1159/000502738 for all online suppl. material).

\section{Results}

In this section, we summarize the simulation results on the estimation of additive interaction. The results on the estimation of other model parameters can be found in online supplementary Figures S5-S20.

\section{The True Model Does Not Have a}

\section{Multiplicative Interaction}

Figure 1 shows the results of simulations where both the true and analysis models are additive, i.e., neither model had a $G \times E$ product term for multiplicative interaction. Thus, the analysis model is the same as the true model generating the data. In general, the offset, IPW, and MI methods all have coverage probability close to that of the full cohort design for estimating additive interaction. All methods have small MSE $(<0.001)$. As expected, the stratified two-phase designs have higher MSE than the full cohort approach, and the MSEs of all methods decrease towards zero as the sample size increases. When the sample size is small, the offset method has slightly smaller MSE than the IPW and MI methods under most settings; however, when $G$ is continuous and
$E$ has a large effect, the MI method has slightly smaller MSE than the offset and IPW methods. Thus, the offset and MI methods have overall good performances for estimating additive interaction compared to the IPW method.

Figure 2 shows the results of simulations where the true model is additive, but a multiplicative interaction term is included in the analysis model (i.e., over-fitting). The offset method has coverage close to that of the full cohort. The MI method does not have good coverage when $E$ has a large effect under continuous $G$. In general, the offset, IPW, and MI methods have similar MSEs. However, for continuous $G$ with $E$ having a large effect, the MI and offset methods have smaller MSEs than the IPW method when the sample size is small.

\section{The True Model Includes a Multiplicative Interaction}

Figure 3 shows the results of simulations where the true model includes a multiplicative interaction term but the analysis model is additive (i.e., under-fitting). All methods, including the full cohort approach, have poor coverage probabilities. This aligns with the large bias noted for all methods (online suppl. material). As before, all methods have MSEs of small magnitude. The MI method has a smaller MSE than the offset and IPW methods, except when $G$ is binary and $E$ has a small effect.

Figure 4 shows the results of simulations where both the true and analysis models include a multiplicative interaction term. The offset method has coverage closer to that of the full cohort analysis. The IPW method performs similar to the offset method, while the MI method has poor coverage. Once again, the magnitude of MSE is small for all methods. The offset and MI methods have smaller MSEs than the IPW method. While the MSEs of the offset, IPW, and MI methods are larger than that of the full cohort analysis for small sample size, the MSEs of all methods decrease towards zero as the sample size increases.

The bias in the estimated additive interaction effect and the SEE decrease with increasing sample size for all methods (online suppl. Fig. S1-S4). There is substantial bias in all methods when the analysis model under-fitted the data, i.e., when the true model includes a multiplicative interaction term but the analysis model is additive (online suppl. Fig. S3). When the analysis model contains a multiplicative interaction term, the MI method has larger bias than the other methods (online suppl. Fig. S2, S4), reflecting the challenges in imputation when the underlying model is not additive. 

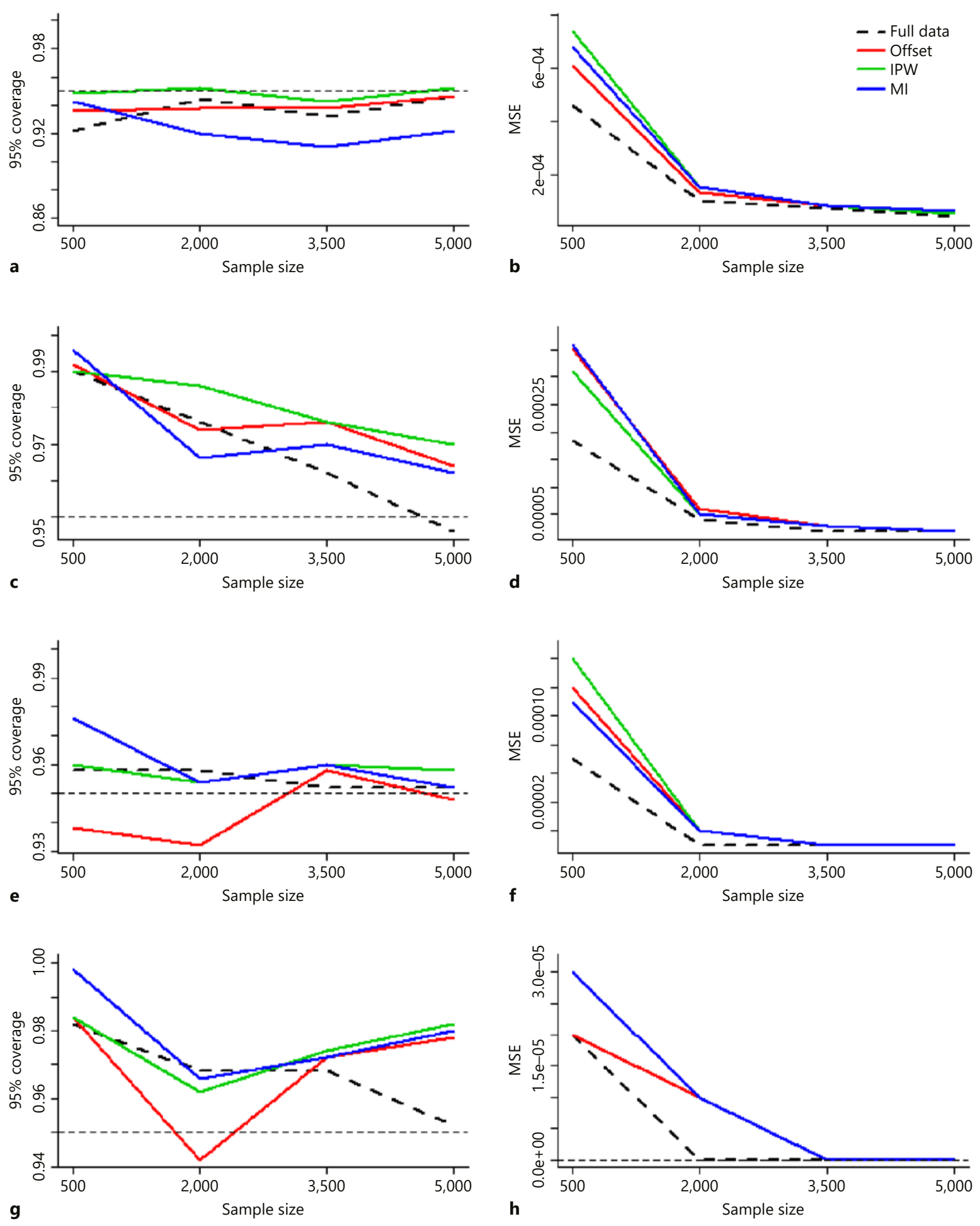

Fig. 1. Simulation results showing the performance of the offset, inverse-probability weighting (IPW), and multiple imputation (MI) methods on additive interaction estimation when both the true and analysis models are additive (i.e., neither contained a $G \times$ $E$ term). a, b $G$ is a binary variable, and $E$ has a large effect. c, d $G$

is a binary variable, and $E$ has a small effect. e, $\mathbf{f} G$ is a continuous variable, and $E$ has a large effect. $\mathbf{g}, \mathbf{h} G$ is a continuous variable, and $E$ has a small effect. a, c, e, $\mathbf{g} 95 \%$ empirical coverage. b, d, f, h Mean squared error (MSE). 

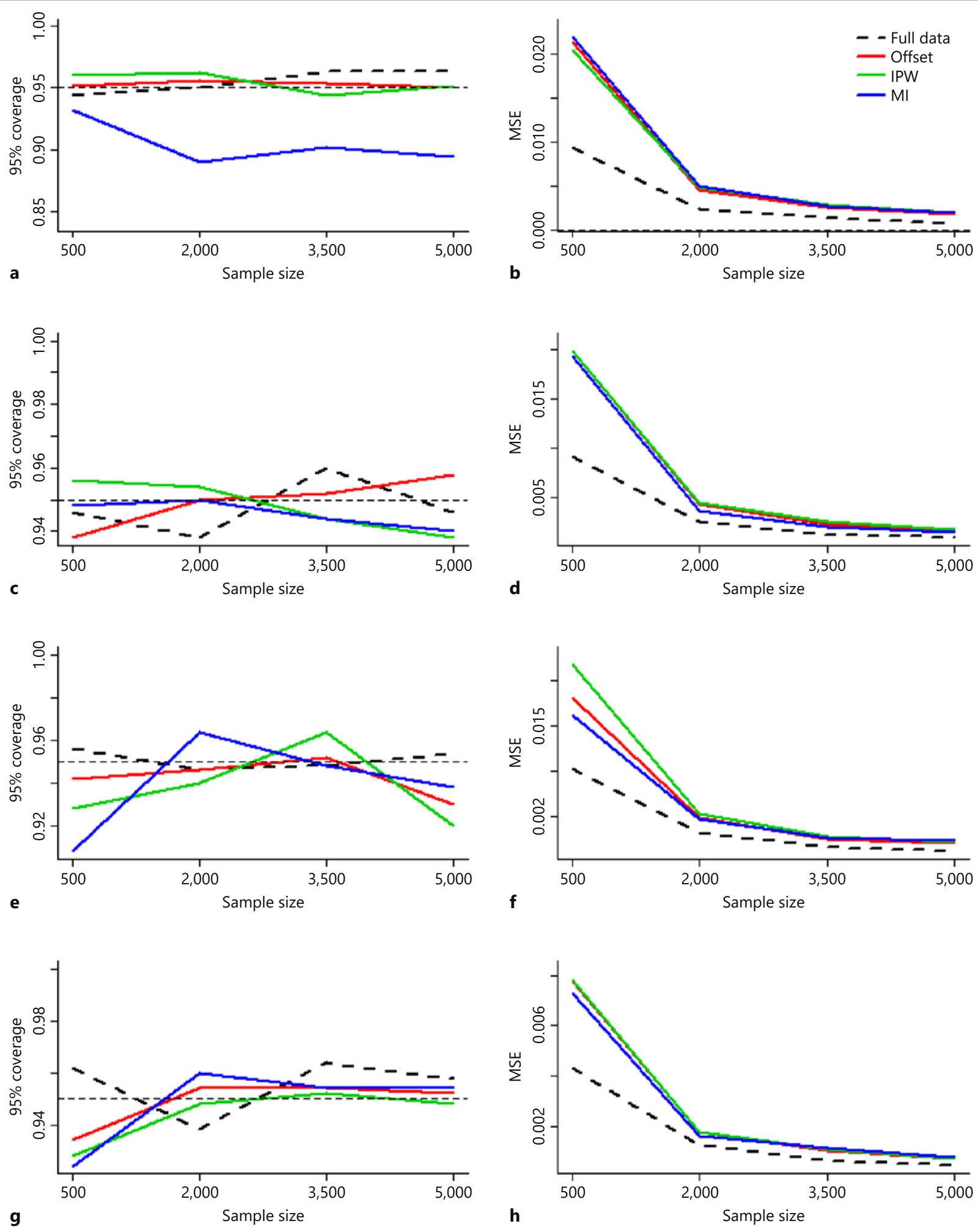

Fig. 2. Simulation results showing the performance of the offset, inverse-probability weighting (IPW), and multiple imputation (MI) methods on additive interaction estimation when the true model is additive but the analysis model has a $G \times E$ term. a, b $G$ is a binary variable, and $E$ has a large effect. c, $\mathbf{d} G$ is a binary variable, and $E$ has a small effect. e, $\mathbf{f} G$ is a continuous variable, and $E$ has a large effect. $\mathbf{g}, \mathbf{h} G$ is a continuous variable, and $E$ has a small effect. a, c, e, g 95\% empirical coverage. b, d, f, h Mean squared error (MSE). 

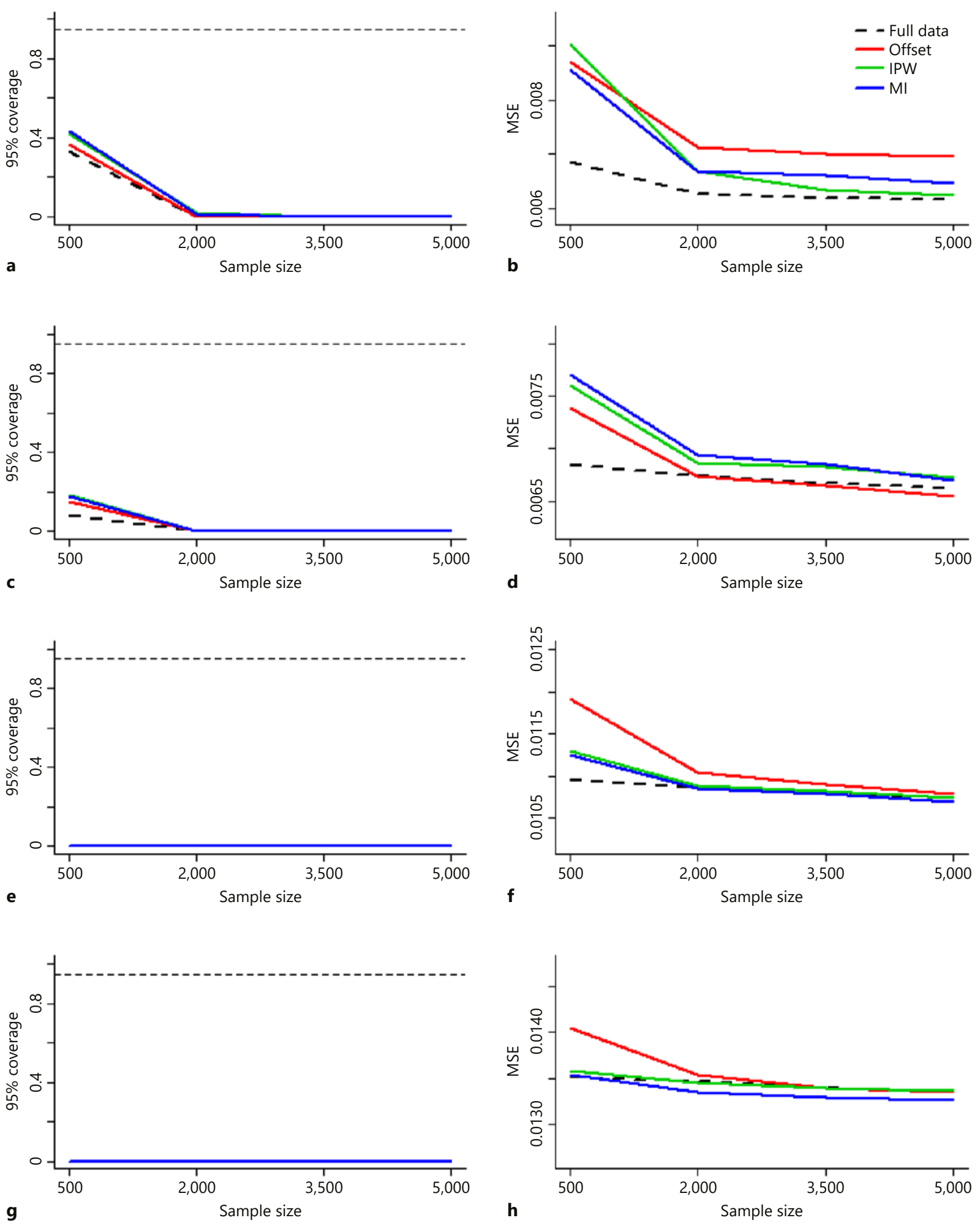

Fig. 3. Simulation results showing the performance of the offset, inverse-probability weighting (IPW), and multiple imputation (MI) methods on additive interaction estimation when the true model has a $G \times E$ term but the analysis model is additive. a, b $G$ is a binary

variable, and $E$ has a large effect. c, $\mathbf{d} G$ is a binary variable, and $E$ has a small effect. e, $\mathbf{f} G$ is a continuous variable, and $E$ has a large effect. $\mathbf{g}, \mathbf{h} G$ is a continuous variable, and $E$ has a small effect. a, c, e, g 95\% empirical coverage. b, d, f, h Mean squared error (MSE). 

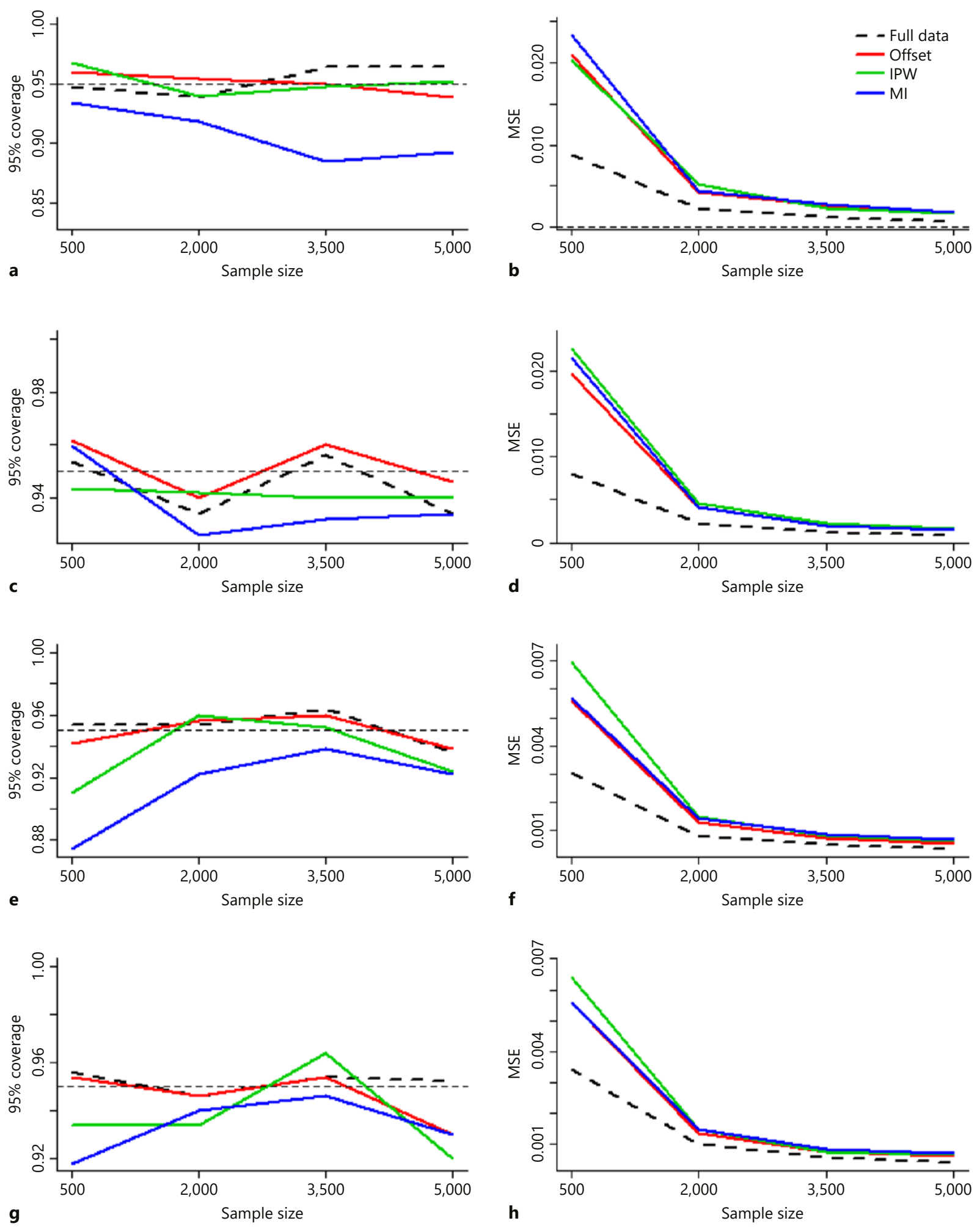

Fig. 4. Simulation results showing the performance of the offset, inverse-probability weighting (IPW), and multiple imputation (MI) methods on additive interaction estimation when both the true model and analysis model have a $G \times E$ term. a, b $G$ is a binary vari- able, and $E$ has a large effect. $\mathbf{c}, \mathbf{d} G$ is a binary variable, and $E$ has a small effect. e, $\mathbf{f} G$ is a continuous variable, and $E$ has a large effect. $\mathbf{g}, \mathbf{h} G$ is a continuous variable, and $E$ has a small effect. a, c, e, $\mathbf{g}$ $95 \%$ empirical coverage. b, d, f, h Mean squared error (MSE). 


\section{Summary of the Results}

Overall, our simulations suggest that the offset method performs well. The MI method performs well when both the true and analysis models are additive. The MI method does not provide a discernible advantage over the offset method when the analysis model includes multiplicative interaction terms. Thus, the MI method may not be the best choice for estimating additive interaction effect when the analysis model includes multiplicative interaction terms; the offset method has the best bias-variance tradeoff (as measured by MSE) for estimating additive interaction in this setting.

\section{Discussion}

In this paper, we have investigated the properties of three different methods for estimating additive interactions and their SEs in stratified two-phase case-controls studies. Our simulations have focused on stratified twophase sampling where the parent cohort is ascertained in a prospective manner. The key to the estimation of additive interactions is to consistently estimate the intercept and the effect of the stratification variable. The offset and IPW methods are among the popular approaches for estimating model parameters under a stratified case-control design [46]. MI tackles the estimation problem from the perspective of missing data [47].

MI has several advantages. It is easy to use and is implemented in most standard statistical packages. The SE estimates under MI can be easily obtained using Rubin's rule [38]. Thus, no additional steps are needed to account for the extra information as well as variability carried in the estimated offsets or weights under a stratified twophase case-control design. Another potential advantage of MI is that, when there are missing data in other covariates, it is straightforward to impute these variables as part of the MI strategy, thereby improving the efficiency of the estimates relative to analyses based on removing individuals with missing values in these covariates. In contrast, the offset and IPW methods would either need an extra step to impute the missing covariates or use only individuals without any missing values in the analyses, resulting in efficiency loss. Last, when some covariates are available for the parent data set, MI can naturally incorporate the additional information in the estimation process, resulting in potentially more efficient estimates than those from the offset and IPW methods.

MI also has its limitations. A critical requirement for the imputation model is that it has to be compatible with the analysis model $[41,44]$. This requirement becomes difficult when the analysis model contains nonlinear terms of the covariates such as multiplicative interaction or polynomials. A systematic treatment of this topic is beyond the scope of this paper. In our simulation studies and the E2C2 data analysis, we accommodated $G \times E$ multiplicative interaction in the analysis model by performing MI separately for each stratum of $G$, thereby allowing the relationship between $E$ and $D$ to vary across $G$. Our simulation results suggest that this strategy is not completely satisfactory, as evidenced by the bias in the estimated additive interaction and suboptimal coverage of the MI method when the analysis model contained multiplicative interaction effect. Based on the findings form the simulation studies and considering the strengths and limitations of the MI method, we recommend using MI to estimate additive interactions under stratified casecontrol samples when there is no multiplicative interaction term in the analysis model. In practice, we suggest a two-step process. First, using a naive logistic regression to determine whether a multiplicative interaction is needed. When there is no evidence of multiplicative interaction, estimate the additive interaction using the MI method; otherwise use the offset method.

The stratified two-phase case-control design considered in this paper can lend itself to any large epidemiology cohort study. One example is the Atherosclerosis Risk in Communities (ARIC) study [48], where a cohort of 15,792 subjects were sampled from 4 US communities and were followed for 10 years for the development of various heart diseases. Numerous variables were collected, and DNA specimen is available for all subjects to obtain genetic information upon request. Since the specimen is in limited quantity, it would not be feasible to request DNA specimen for the entire cohort to conduct a gene-environment interaction study. Case-control or other similar designs are ideal choices in this situation. A stratified case-cohort study has been conducted on ARIC data to identify risk factors of incident coronary heart disease [49]. It would be interesting to study an additive gene-environment interaction effect on risks of various heart diseases using ARIC data with a two-phase stratified case-control design. The estimation methods investigated in this paper can be applied to such studies.

The stratification used in the design ensures balanced numbers of cases and controls in each stratum. As a result, it avoids potential efficiency loss due to highly unbalanced distribution of the stratification variable between sampled cases and controls. This design is similar to the exposure enriched sampling designs $[50,51]$ in 
that phase two sampling is stratified by one of the risk factors of interest (e.g., exposure factor) with stratumspecific sampling probabilities. However, they differ in that the design in this paper aims to oversample cases and achieve balance between cases and controls in each stratum, whereas the latter design aims to oversample the rare exposure strata. It would be interesting to study how an outcome- and exposure-enriched sampling design would affect the efficiency for estimating additive interactions.

Some existing large epidemiology studies have ascertained risk factors using a population-based stratified two-phase case-control sampling approach. An example is the US Kidney Cancer Study, a population-based stratified case-control study, of the association between hypertension and renal cell carcinoma in black and white men and women [52]. The design considered in this paper is different from the population-based stratified case-control (PBSCC) design [53]. Among many differences between them, two have important implications on additive interaction estimation. First, the marginal disease probability that is needed to estimate the additive interaction can be easily estimated in the twophase design when a prospectively sampled parent cohort is available in phase one. In PBSCC, the marginal disease probability is more difficult to estimate since the controls are selected directly from the population, whose size is typically unknown and, therefore, needs to be estimated under possibly complex sampling scheme. The potential sample frame deficiency can also lead to a biased estimate of the population size. Second, stratified simple random sampling is used to select controls from the parent cohort in the two-phase design, whereas clustered sampling could be used in PBSCC to choose controls from the population, which complicates the variance estimation of the additive interaction due to the intra-cluster correlation among subjects. The methods studied in this paper can be potentially extended to the PBSCC design provided that the stratum-specific marginal disease probability can be reliably estimated, and the intra-cluster correlation is properly accounted for in the variance estimation.

Several extensions of our work are possible. In this paper, we focused on estimating additive interaction effects and their SEs. We have not examined hypothesis tests, power, and type I errors. These properties will be examined in future works and compared with other existing inferential procedures for additive interactions. There is a strong line of research on efficient estimation of gene-environment interaction in case-control studies exploiting the assumption of gene-environment independence $[19,54-59]$. These studies have focused on population-based case-control design and/or assumed rare disease. It would be interesting to extend these methods to the estimation of additive interaction under a stratified two-phase case-control design for non-rare diseases. Another extension is to consider time to event outcome. Using a survival analysis framework, the analogous design is the nested case-control design [60], where all individuals with events are sampled and, at each event time, a person from the risk set is randomly sampled and maybe matched to the corresponding event by genetic or environmental factors. There are two potential modeling strategies for nested case-control design - proportional hazards model and additive hazards model [61]. In either of these models, one needs to estimate the main effect of the matching variables and the baseline hazards function to obtain an estimate of the additive interaction. We will investigate these methods in future work.

The $\mathrm{R}$ code implementing the estimation methods investigated in this paper is available from the authors upon request.

\section{Acknowledgments}

We thank Dr. Allan Halpern for providing us access to data from the study of nevi in children. This work was supported through grants P30 CA008748, R01 CA137420, and R01 CA197402 from the National Cancer Institute, USA, and grant UL1RR024996 from the Clinical and Translational Science Center at Weill Cornell Medicine, New York, USA. The content is solely the responsibility of the authors and does not represent the official views of the National Institutes of Health. The majority of the work in this paper was done when $\mathrm{Ai} \mathrm{Ni}$ was at the Department of Epidemiology and Biostatistics, Memorial Sloan Kettering Cancer Center, New York, USA.

\section{Statement of Ethics}

The two epidemiology studies used in this paper were approved by the corresponding Institutional Review Boards and informed consent was obtained from all participants.

\section{Disclosure Statement}

The authors declare no conflicts of interest. 


\section{Appendix}

Derivation of Variance Estimator in the Offset Method

In essence, including estimated offsets in likelihood function 4, when estimating regression coefficients, is equivalent to jointly estimating the distribution parameters of some functions of the offsets and the regression coefficients. Define $\eta_{h 1}$ as the true probability of a case being in stratum $h$ and define $\eta_{h 0}$ similarly for controls. Thus $\eta_{h d}(d=0,1)$ is the parameter vector of a multinomial distribution. The offsets can be written as $\log \left(n_{h 1} N_{0} \eta_{h 0} / n_{h 0} N_{1} \eta_{h 1}\right)$. Then, log-likelihood function 5 can be reparameterized for parameters $\eta$ and $\beta$ and written as

$$
\ell(\eta, \beta)=\sum_{h=1}^{H} \sum_{i=1}^{n_{h}} \log \left\{\operatorname{pr}\left(D_{h i} \mid S_{h i}=1, Z_{h i} ; \eta, \beta\right)\right\} .
$$

Let $(\bar{\beta}, \bar{\eta})$ and $(\hat{\beta}, \hat{\eta})$ be the true values and maximum likelihood estimates of $(\beta, \eta)$, respectively. By Taylor's expansion of the first partial derivative of the log-likelihood at the true parameters, we get

$$
\begin{aligned}
& \frac{\partial \ell(\hat{\eta}, \hat{\beta})}{\partial \beta}=\frac{\partial \ell(\bar{\eta}, \bar{\beta})}{\partial \beta}+(\hat{\beta}-\bar{\beta})^{T} \frac{\partial^{2} \ell(\bar{\eta}, \bar{\beta})}{\partial \beta \partial \beta^{T}}+ \\
& (\hat{\eta}-\bar{\eta})^{T} \frac{\partial^{2} \ell(\bar{\eta}, \bar{\beta})}{\partial \beta \partial \eta^{T}}+o_{p}(\hat{\beta}-\bar{\beta})+o_{p}(\hat{\eta}-\bar{\eta}) .
\end{aligned}
$$

Using the fact that $\partial \ell(\hat{\eta}, \hat{\beta}) / \partial \beta=0$, we have that

$$
\begin{aligned}
& \operatorname{var}(\hat{\beta})= \\
& \left\{\frac{\partial^{2} \ell(\bar{\eta}, \bar{\beta})}{\partial \beta \partial \beta^{T}}\right\}^{-1}\left[\operatorname{var}\left\{\frac{\partial \ell(\bar{\eta}, \bar{\beta})}{\partial \beta}\right\}+\frac{\partial^{2} \ell(\bar{\eta}, \bar{\beta})}{\partial \beta \partial \eta^{T}}\right. \\
& \left.\operatorname{var}(\hat{\eta})\left\{\frac{\partial^{2} \ell(\bar{\eta}, \bar{\beta})}{\partial \beta \partial \eta^{T}}\right\}^{T}\right]\left\{\frac{\partial^{2} \ell(\bar{\eta}, \bar{\beta})}{\partial \beta \partial \beta^{T}}\right\}^{-1} .
\end{aligned}
$$

By the central limit theorem, $n^{-1 / 2} \partial \ell(\bar{\eta}, \bar{\beta}) / \partial \beta$ converges to a normal distribution with mean zero and covariance matrix

$$
\begin{aligned}
& E\{\partial \ell(\bar{\eta}, \bar{\beta}) / \partial \beta\}\left\{\partial \ell(\bar{\eta}, \bar{\beta}) / \partial \beta^{T}\right\}- \\
& E\{\partial \ell(\bar{\eta}, \bar{\beta}) / \partial \beta\} E\left\{\partial \ell(\bar{\eta}, \bar{\beta}) / \partial \beta^{T}\right\} .
\end{aligned}
$$

Let $\bar{\eta}_{d}=\left(\bar{\eta}_{1 d}, \ldots, \bar{\eta}_{H d}\right)$ and $\hat{\eta}_{d}=\left(\hat{\eta}_{1 d}, \ldots, \hat{\eta}_{H d}\right)$ with $d=0$ for controls and $d=1$ for cases. Then, $n^{1 / 2}\left(\hat{\eta}_{d}-\bar{\eta}_{d}\right)$ converges to a normal distribution with mean zero and covariance matrix $\left(q_{i j}\right)$, where $q_{i j}=\bar{\eta}_{i d}\left(1-\bar{\eta}_{i d}\right)$ if $i=j$ and $q_{i j}=-\bar{\eta}_{i d} \bar{\eta}_{j d}$ if $i \neq j$. Plug in the estimates of these covariance matrices to equation 10 and with some algebra we arrive at

$$
\operatorname{var}(\hat{\beta})=\left(Z^{T} V Z\right)^{-1}\left\{Z^{T} V Z-C\right\}\left(Z^{T} V Z\right)^{-1},
$$

where $Z$ is the design matrix, $V$ is an $n \times n$ diagonal matrix with element $v_{h i}=\hat{p}_{h i}\left(1-\hat{p}_{h i}\right)$ with $\hat{p}_{h i}$ being the estimated disease probability for subject $i$ in stratum $h$,

$$
C=\sum_{h=1}^{H}\left(\frac{1}{n_{h 0}}-\frac{1}{N_{h 0}}+\frac{1}{n_{h 1}}-\frac{1}{N_{h 1}}\right) W_{h} W_{h}^{T}+\left(\frac{1}{N_{0}}+\frac{1}{N_{1}}\right) W W^{T},
$$

where $W_{h}=X^{T} V e_{h}$ with $e_{h}$ being an $n \times 1$ vector with ones in locations corresponding to the $h$-th stratum and zeros elsewhere, and

$$
W=\sum_{h=1}^{H} W_{h} .
$$

\section{Appendix Table A1}

Numbers of Cases and Controls in the 14 SSI Strata in the SONIC Study

\begin{tabular}{lccc}
\hline Strata & Case & Control & Total \\
\hline 1, SSI $\in[0,0.2)$ & 4 & 22 & 26 \\
2, SSI $\in[0.2,0.4)$ & 6 & 14 & 20 \\
3, SSI $\in[0.4,0.6)$ & 12 & 17 & 29 \\
4, SSI $\in[0.6,0.8)$ & 14 & 10 & 24 \\
5, SSI $\in[0.8,1.0)$ & 3 & 4 & 7 \\
6, SSI $\in[1.0,1.2)$ & 5 & 15 & 20 \\
7, SSI $\in[1.2,1.4)$ & 7 & 24 & 31 \\
\hline 8, SSI $\in[1.4,1.6)$ & 14 & 54 & 68 \\
9, SSI $\in[1.6,1.8)$ & 17 & 59 & 76 \\
10, SSI $\in[1.8,2.0)$ & 5 & 20 & 25 \\
11, SSI $\in[2.0,2.2)$ & 1 & 8 & 9 \\
12, SSI $\in[2.2,2.4)$ & 1 & 30 & 31 \\
13, SSI $\in[2.4,2.6)$ & 1 & 24 & 25 \\
14, SSI $\in[2.6,3.0]$ & 1 & 51 & 52 \\
\hline Total & 91 & 352 & 443 \\
\hline
\end{tabular}

SSI, sun sensitivity index.

\section{References}

1 Neyman J. Contribution to the theory of sampling human populations. J Am Stat Assoc. 1938;33(201):101-16.

2 Scott AJ, Wild CJ. Fitting regression models to case-control data by maximum likelihood. Biometrika. 1997;84(1):57-71.

3 Breslow NE, Chatterjee N. Design and analysis of two-phase studies with binary outcome applied to wilms tumour prognosis. J R Stat Soc Ser C Appl Stat. 1999;48(4):457-68.
4 White JE. A two stage design for the study of the relationship between a rare exposure and a rare disease. Am J Epidemiol. 1982 Jan; 115(1):119-28

5 Legg JC, Fuller WA. Two-phase sampling. Handbook of statistics. Volume 29. Oxford: Elsevier; 2009. pp. 55-70.

6 Moslehi R, Chatterjee N, Church TR, Chen J, Yeager M, Weissfeld J, et al. Cigarette smoking, $\mathrm{N}$-acetyltransferase genes and the risk of advanced colorectal adenoma. Pharmacogenomics. 2006 Sep;7(6):819-29. 
7 Setiawan VW, Doherty JA, Shu XO, Akbari MR, Chen C, De Vivo I, et al. Two estrogenrelated variants in CYP19A1 and endometrial cancer risk: a pooled analysis in the Epidemiology of Endometrial Cancer Consortium. Cancer Epidemiol Biomarkers Prev. 2009 Jan; 18(1):242-7.

8 Cordell HJ. Detecting gene-gene interactions that underlie human diseases. Nat Rev Genet. 2009 Jun;10(6):392-404.

9 Izumi S, Sakata R, Yamada M, Cologne J. Interaction between a single exposure and age in cohort-based hazard rate models impacted the statistical distribution of age at onset. J Clin Epidemiol. 2016 Mar;71:43-50.

10 Thomas D. Methods for investigating geneenvironment interactions in candidate pathway and genome-wide association studies. Annu Rev Public Health. 2010;31(1):21-36.

11 Elston RC. On additivity in the analysis of variance. Biometrics. 1961;17(2):209-19.

12 Rothman KJ, Greenland S, Lash TL. Modern epidemiology. Philadelphia: Lippincott, Williams and Wilkins; 2008.

13 Begg CB, Zhang ZF. Statistical analysis of molecular epidemiology studies employing caseseries. Cancer Epidemiol Biomarkers Prev. $1994 \mathrm{Mar} ; 3(2): 173-5$.

14 Kalilani L, Atashili J. Measuring additive interaction using odds ratios. Epidemiol Perspect Innov. 2006 Apr;3(1):5.

15 Karlson EW, Chang SC, Cui J, Chibnik LB, Fraser PA, De Vivo I, et al. Gene-environment interaction between hla-drb1 shared epitope and heavy cigarette smoking in predicting incident rheumatoid arthritis. Ann Rheum Dis. 2010 Jan;69(1):54-60.

16 Kiyohara C, Washio M. The role of geneenvironment interaction in the etiology of SLE. Epidemiological studies of specified rare and intractable disease. Singapore: Springer; 2019. pp. 147-62.

17 Meidtner K, Podmore C, Kröger J, van der Schouw YT, Bendinelli B, Agnoli C, et al. Interaction of dietary and genetic factors influencing body iron status and risk of type 2 diabetes within the epic-interact study. Diabetes Care. 2018 Feb;41(2):277-85.

18 Zou GY. On the estimation of additive interaction by use of the four-by-two table and beyond. Am J Epidemiol. 2008 Jul;168(2):21224.

19 Han SS, Rosenberg PS, Garcia-Closas M, Figueroa JD, Silverman D, Chanock SJ, et al. Likelihood ratio test for detecting gene $(\mathrm{G})$ environment $(\mathrm{E})$ interactions under an additive risk model exploiting G-E independence for case-control data. Am J Epidemiol. 2012 Dec;176(11):1060-7.

20 Flanders WD, Greenland S. Analytic methods for two-stage case-control studies and other stratified designs. Stat Med. 1991;10(5):739_ 47.

21 Korn EL, Graubard BI. Analysis of large health surveys: accounting for the sampling design. J R Stat Soc Ser A Stat Soc. 1995; 158(2):263-95.
22 Breslow NE, Holubkov R. Maximum likelihood estimation of logistic regression parameters under two-phase, outcome-dependent sampling. J R Stat Soc Series B Stat Methodol. 1997;59(2):447-61.

23 Chatterjee N, Chen YH, Breslow NE. A pseudoscore estimator for regression problems with two-phase sampling. J Am Stat Assoc. 2003;98(461):158-68.

24 Lee A, Scott A, Wild C. Efficient estimation in multi-phase case-control studies. Biometrika. 2010;97(2):361-74.

25 Tao R, Zeng D, Lin DY. Efficient semiparametric inference under two-phase sampling, with applications to genetic association studies. J Am Stat Assoc. 2017;112(520):1468-76.

26 Amorim G, Alastair S, Wild CJ. Multi-phase sampling. Handbook of statistical methods for case-control studies. Boca Raton: CRC Press; 2018

27 Espin-Garcia O, Craiu RV, Bull SB. Twophase designs for joint quantitative-traitdependent and genotype-dependent sampling in post-GWAS regional sequencing. Genet Epidemiol. 2018 Feb;42(1):104-16.

28 Shen C. Application of multiple imputation to data from two-phase sampling: estimation of the incidence rate of cognitive impairment. J Data Sci. 2007;5(4):503-18.

29 Marti H, Chavance M. Multiple imputation analysis of case-cohort studies. Stat Med. 2011 Jun;30(13):1595-607.

30 Enders D, Kollhorst B, Engel S, Linder R, Pigeot I. Comparison of multiple imputation and two-phase logistic regression to analyse two-phase case-control studies with rich phase 1: a simulation study. J Stat Comput Simul. 2018;88(11):2201-14.

31 Oliveria SA, Satagopan JM, Geller AC, Dusza SW, Weinstock MA, Berwick M, et al. Study of Nevi in Children (SONIC): baseline findings and predictors of nevus count. Am J Epidemiol. 2009 Jan;169(1):41-53.

$32 \mathrm{Xu} \mathrm{H}$, Marchetti MA, Dusza SW, Chung E, Fonseca M, Scope A, et al. Factors in Early Adolescence Associated With a Mole-Prone Phenotype in Late Adolescence. JAMA Dermatol. 2017 Oct;153(10):990-8.

33 Agresti A. Categorical data analysis. Volume 482. Hoboken: John Wiley \& Sons; 2003.

34 Wang X, Elston RC, Zhu X. The meaning of interaction. Hum Hered. 2010;70(4):269-77.

35 Breslow N, Cain K. Logistic regression for two-stage case-control data. Biometrika. 1988;75(1):11-20.

36 Breslow N, Zhao L, Fears TR, Brown CC. Logistic regression for stratified case-control studies. Biometrics. 1988 Sep;44(3):891-9.

37 Hsieh DA, Manski CF, McFadden D. Estimation of response probabilities from augmented retrospective observations. J Am Stat Assoc. 1985;80(391):651-62.

38 Rubin DB. Multiple imputation for nonresponse in surveys. Volume 81. New York: John Wiley \& Sons; 1987.
39 Schenker N, Taylor JMG. Partially parametric techniques for multiple imputation. Comput Stat Data Anal. 1996;22(4):425-46.

40 Lavori PW, Dawson R, Shera D. A multiple imputation strategy for clinical trials with truncation of patient data. Stat Med. 1995 Sep; 14(17):1913-25.

41 Schafer JL. Analysis of incomplete multivariate data. Boca Raton: CRC Press; 1997.

42 van Buuren S, Groothuis-Oudshoorn K. mice: multivariate imputation by chained equations in R. J Stat Softw. 2011;45(3):1-67.

43 Moons KG, Donders RA, Stijnen T, Harrell FE Jr. Using the outcome for imputation of missing predictor values was preferred. J Clin Epidemiol. 2006 Oct;59(10):1092-101.

44 Rubin DB. Multiple imputation after 18+ years. J Am Stat Assoc. 1996;91(434):473-89.

45 Olson SH, Chen C, De Vivo I, Doherty JA, Hartmuller V, Horn-Ross PL, et al. Maximizing resources to study an uncommon cancer: E2C2 - Epidemiology of Endometrial Cancer Consortium. Cancer Causes Control. 2009 May;20(4):491-6.

46 Whittemore AS. Multistage sampling designs and estimating equations. J R Stat Soc Series B Stat Methodol. 1997;59(3):589-602.

47 Little RJA, Rubin DB. Statistical analysis with missing data. Volume 333. Hoboken: John Wiley \& Sons; 2014.

48 The Atherosclerosis Risk in Communities (ARIC) study: design and objectives. The ARIC investigators. Am J Epidemiol. 1989 Apr;129(4):687-702.

49 Ballantyne CM, Hoogeveen RC, Bang $\mathrm{H}$, Coresh J, Folsom AR, Heiss G, et al. Lipoprotein-associated phospholipase A2, high-sensitivity C-reactive protein, and risk for incident coronary heart disease in middle-aged men and women in the Atherosclerosis Risk in Communities (ARIC) study. Circulation. 2004 Feb;109(7):837-42.

50 Stenzel SL, Ahn J, Boonstra PS, Gruber SB, Mukherjee B. The impact of exposure-biased sampling designs on detection of gene-environment interactions in case-control studies with potential exposure misclassification. Eur J Epidemiol. 2015 May;30(5):413-23.

51 Sun Z, Mukherjee B, Estes JP, Vokonas PS, Park SK. Exposure enriched outcome dependent designs for longitudinal studies of geneenvironment interaction. Stat Med. 2017 Aug; 36(18):2947-60.

52 Colt JS, Schwartz K, Graubard BI, Davis F, Ruterbusch J, DiGaetano R, et al. Hypertension and risk of renal cell carcinoma among white and black Americans. Epidemiology. 2011 Nov;22(6):797-804.

53 Hancock DB, Scott WK. Population-based case-control association studies. Curr Protoc Hum Genet. 2012 Jul;1(1):17.

54 Piegorsch WW, Weinberg CR, Taylor JA. Non-hierarchical logistic models and caseonly designs for assessing susceptibility in population-based case-control studies. Stat Med. 1994 Jan;13(2):153-62. 
55 Umbach DM, Weinberg CR. Designing and analysing case-control studies to exploit independence of genotype and exposure. Stat Med. 1997 Aug;16(15):1731-43.

56 Chatterjee N, Carroll RJ. Semiparametric maximum likelihood estimation exploiting gene-environment independence in casecontrol studies. Biometrika. 2005;92(2):399418.

57 Mukherjee B, Chatterjee N. Exploiting gene-environment independence for analysis of case- control studies: an empirical Bayes-type shrinkage estimator to trade-off between bias and efficiency. Biometrics. 2008 Sep;64(3):685-94.

58 Chen J, Kang G, Vanderweele T, Zhang C, Mukherjee B. Efficient designs of gene-environment interaction studies: implications of Hardy-Weinberg equilibrium and gene-environment independence. Stat Med. 2012 Sep; 31(22):2516-30.

59 Liu G, Mukherjee B, Lee S, Lee AW, Wu AH, Bandera EV, et al.; Ovarian Cancer Associa- tion Consortium. Robust tests for additive gene-environment interaction in case-control studies using gene-environment independence. Am J Epidemiol. 2018 Feb;187(2): 366-77.

60 Thomas D. Addendum to: methods of cohort analysis: appraisal by application to asbestos mining. J R Stat Soc [Ser A]. 1977;140:469-91.

61 Lin D, Ying Z. Semiparametric analysis of the additive risk model. Biometrika. 1994;81(1): 61-71. 